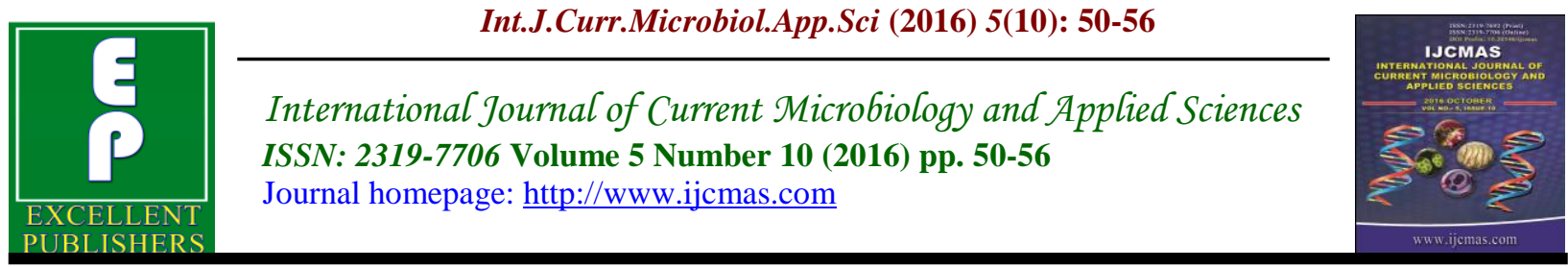

Original Research Article

http://dx.doi.org/10.20546/ijcmas.2016.510.007

\title{
Green Synthesis of Silver Nano Particles from Aspergillus niger (MTCC-961)
}

\author{
P. Kalyani, K. Chandana Vineela, S. Geetha* and K.P.J. Hemalatha \\ Department of Microbiology, Andhra University Visakhapatnam -530 003, India \\ *Corresponding author email id:
}

Keywords

Ag-NPs, A. niger,

UV- visible

spectrophotometer,

FTIR,

Antibacterial

activity.

\section{Article Info}

Accepted:

12 September 2016

Available Online:

10 October 2016

\section{A B S T R A C T}

The silver nanoparticles synthesized biologically have been widely used in medicinal field. The synthesis of silver nanoparticles has been carried out by using the extracellular filtrate of the fungal strain. In this study, silver nanoparticles were synthesized using the fungus Aspergillus niger with an aqueous solution of $\mathrm{AgNO}_{3}$. Synthesized silver nanoparticles (Ag-NPs) were characterized through UV-visible spectrophotometer and Fourier Transform Infrared Spectroscopy (FTIR). Maximum absorbance was observed at $420 \mathrm{~nm}$ in visible region corresponding to the Plasmon resonance of silver nanoparticles, thus, confirming their presence. The Fourier transform infrared spectroscopy confirmed the presence of protein as stabilizing agent surrounding the silver nanoparticles. The reduction of silver ions was due to amino groups of proteins and other functional groups in the cell free filtrate of fungi. The reduction of silver ions leads to the formation of stable protein capped silver nanoparticles. The Ag-NPs and Ag-NPs + Chloramphenicol (Ab) possess potential antimicrobial activity against to Escherichia coli, Klebsiella pneumonia, Staphylococcus aureus and Streptococcus sp.

\section{Introduction}

In last few years, research in nanotechnology has been carried out extensively as nanoparticles possess increased structural integrity as well as unique chemical, mechanical, optical, electronic and magnetic properties compared to large particles of bulk materials (Bhushan, 2004). In the synthesis of nano particles, the various conventional processes like a number of chemical and physical methods including chemical reduction in aqueous or non-aqueous solution, micro emulsion, sono-chemical and microwave- assisted methods have been applied (Petit et al., 1993, Solanki \& Murthy 2010, Pol et al., 2002 and Wang et al., 2010). Since the chemicals like organic solvents, hydrazine, sodium borohydride and $\mathrm{N}$, N-dimethyl formamide utilized in the synthesis of metal nano particles are found to be highly reactive and biological hazardous, the chemically synthesized metal nano particles could not accomplished with the biomedical field. On the other hand, the microbiologically synthesized metal nanoparticles are found to be eco-friendly, 
reliable, biocompatible and economic. Nanoparticles are synthesized from various microbes through extracellular or intracellular processes (Ravendran et al., 2003). A large number of bacterial and fungal species have antimicrobial activity through reducing the metal ions for production of metal nanoparticles. Recent studies declaring that the fungi showed the good potential for synthesizing bio active compounds when compared with bacteria. This indicates that the fungi were most suitable for production in large amounts (Narayanan and Sakthivel, 2010). In addition, the extracellular biosynthesis using fungi could also make downstream processing much easier (Slawson et al., 2008), high tolerance to temperature fluctuations and show high capacity towards bioaccumulation than bacteria. Accordingly microorganisms such as fungi, bacteria, actinomycetes] and plants have been used for the study of biosynthesis of silver nanoparticles (Mohanpuria et al., 2008; Bhaina, 2006; Binupyiya, 2010; Shahverdi, 2002; Ahmad et al., 2002; Rai et al., 2006 and Sathish kumar et al., 2009).

Synthesis of nanoparticles occurs intracellularly or extracellularly of which the extracellular process could be used preferably as it is less laborious and also is less costly. Research on silver compounds grows to a wide range in order to improve antimicrobial activity, disinfection property. The Ag-NPs gained potential importance because of their salient features like antimicrobial, antisepsis activity they are widely used in medicinal applications and in to maintain the hygiene conditions (Gong et al., 2007). The Ag-NPs have proved that they are more potential effective against bacterial species, viruses and to other eukaryotic microbes.

\section{Materials and Methods}

\section{Culture collection and maintenance}

Pure cultures of Aspergillus niger (MTCC No-961) were purchased from MTCC, Chandigarh, India and were immediately transferred to sterile agar slants of potato dextrose agar media. The strains were grown in potato dextrose media. The Asperigillus niger culture from potato dextrose broth was streaked on a Potato dextrose agar slant and it was incubated at $27^{\circ} \mathrm{C}$ for 72 hours. It was then sub cultured and was stored in refrigerator for further use.

\section{Extracellular Synthesis of Ag-NPs}

The fungal strain Aspergillus niger was freshly inoculated on a potato dextrose broth in flask The flak was incubated on orbital shaker at $30^{\circ} \mathrm{C}$ and agitated at $150 \mathrm{rpm}$ at 3 days. The fungal biomass was harvested after 3 days, by sieving through Whatman No 1 filter paper, later thoroughly wash with deionized water to remove the other components in the media from the biomass. Typically $20 \mathrm{~g}$ of fresh and clean biomass was taken into Erlenmeyer flaks containing $200 \mathrm{ml}$ of deionized water and the flask was incubated at $30^{\circ} \mathrm{C}$ for 3 days and agitated at $150 \mathrm{rpm}$. Later the cell filtrate was obtained through passage of culture media through Whatman No-1 filter paper. Fifty milliliters of cell free filtrate (CFF) was taken into 250 $\mathrm{ml}$ of Erlenmeyer flask and mixed with 1 $\mathrm{mM} \mathrm{AgNO}_{3}\left(0.017 \mathrm{~g} \quad \mathrm{AgNO}_{3} / 100 \mathrm{ml}\right)$ as final concentration. The flasks were incubated at $30^{\circ} \mathrm{C}$ in dark room up to 3 days. Control was maintained (without addition of $\mathrm{AgNO}_{3}$, only cell filtrate) with the experimental flask. In order to usage for future experiments the brownish yellow color Ag- NPs solution was stored in amber color bottles. 


\section{Characterization of Ag-NP}

The synthesized Ag-NPs were first characterized by UV-Visible spectrophotometer in the range of $320-560$ $\mathrm{nm}$ with control as the reference. The surface plasmon resonance peaks are found noted to be reliably around $420 \mathrm{~nm}$ regions further the Ag-NPs kept at room temperature for three months to test their stability. Analysis of Ag-NPs by FTIR through spectrum scanning range $450-4000 \mathrm{~cm}^{-1}$ at resolution of $4 \mathrm{~cm}^{-1}$ was carried out.

\section{Characterization of Antibacterial Activity}

Antibacterial property was performed by using the "Nathan's Agar Well Diffusion" technique. Eight millimeter diameter of 2 wells was made on PDA plates. These PDA plates were inoculated by swabbing the 1824hrs isolates Escherichia coli, Klebsiella pneumonia, Bacillus cereus, Staphylococcus aureus and Streptococcus sp., suspensions in order to get proper growth. The Ag-NPs $(100 \mu \mathrm{l})$ was loaded on one well and other well with $60 \mu \mathrm{l}$ of Ag-NPs $+40 \mu$ of
Chloramphenicol (a wide range of antibacterial drug) each well. Wells without the extracts were maintained as control. After completion of incubation period at $30^{\circ} \mathrm{C}$ temperature and $24 \mathrm{hrs}$ the susceptibility was measured by considering the inhibition zone diameter around each well to the nearest $\mathrm{mm}$.

\section{Results and Discussion}

\section{Ag-Nps synthesis}

The present research work was carried out in the extracellular synthesis Ag- NP in a comprehensive manner. After 3 days of incubation, the fungal biomass was filtered, filtrate was exposed to $\mathrm{AgNO}$. The reaction was started after 24 hours incubation in dark condition, the pale yellow color of the cell free filtrate (CFF) changed to dark brownish yellow color indicating the formation of AgNPs (Fig 1) which correlates with the results obtained by Ingle et al., (2008) and Prameela et al., (2013). There is no color change noted in the control flask incubated in the same environment.

Fig.1 SEM images of Nanoparticles
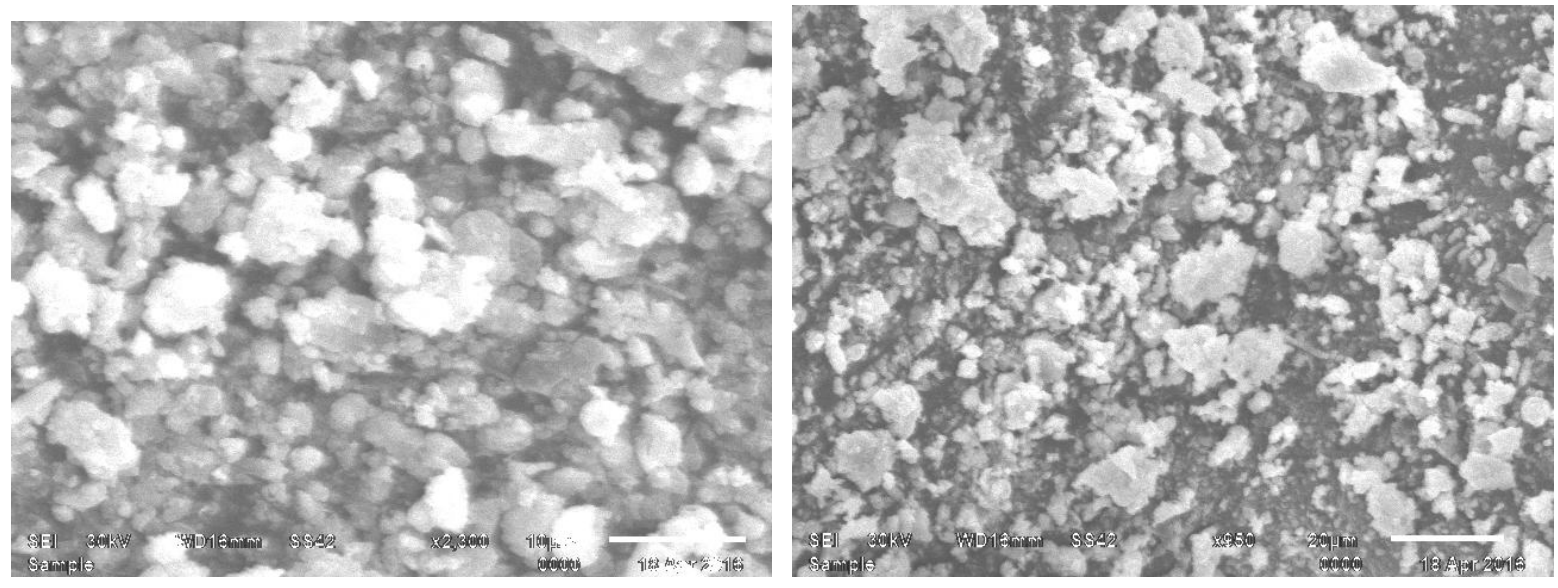
Fig.2 UV-visible spectra of Ag-NPs synthesized by Aspergillus niger

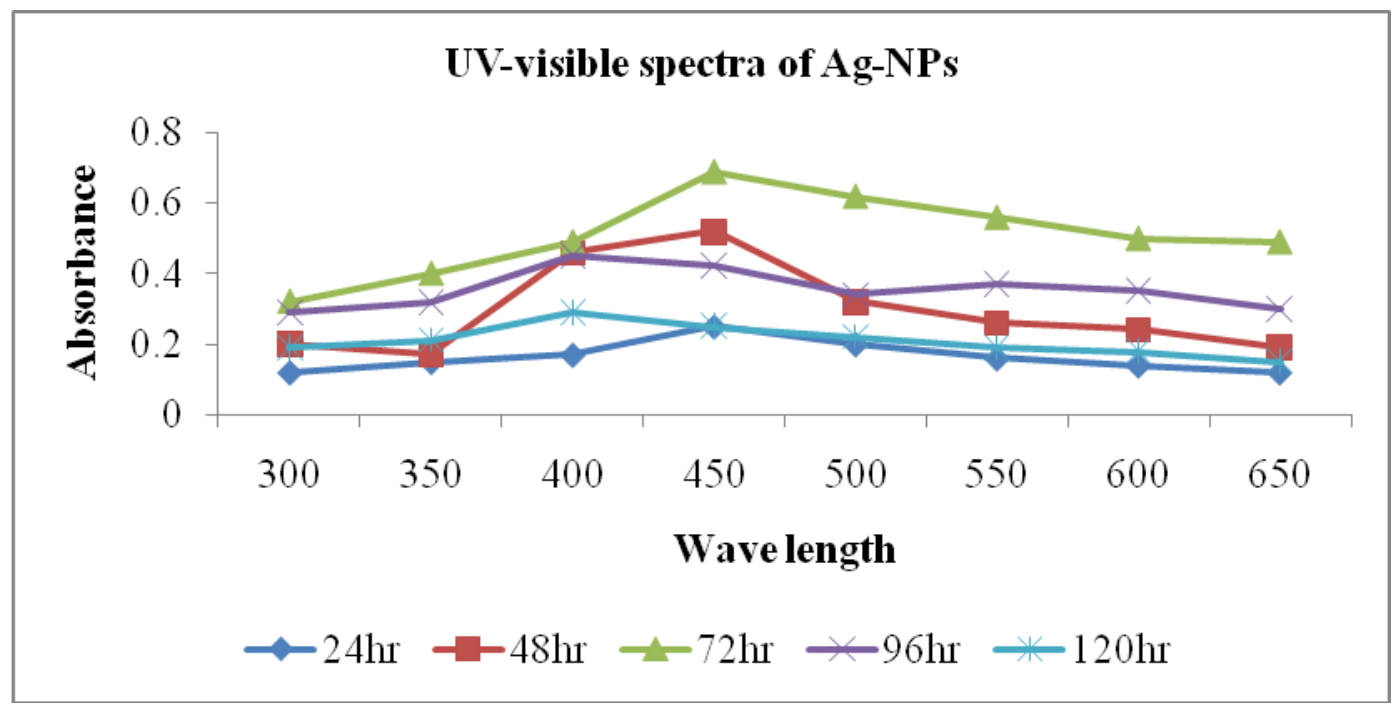

Fig.3 FTIR analysis of Ag-NPs

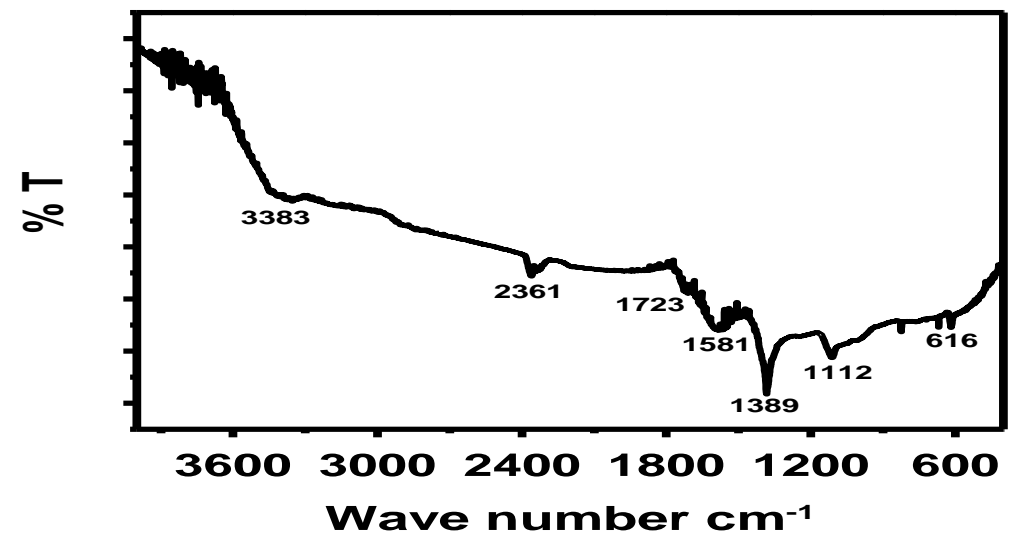

Fig.4 Antibacterial activity of Ag-NPs and Ag-NPs + Chloramphenicol (Ab)

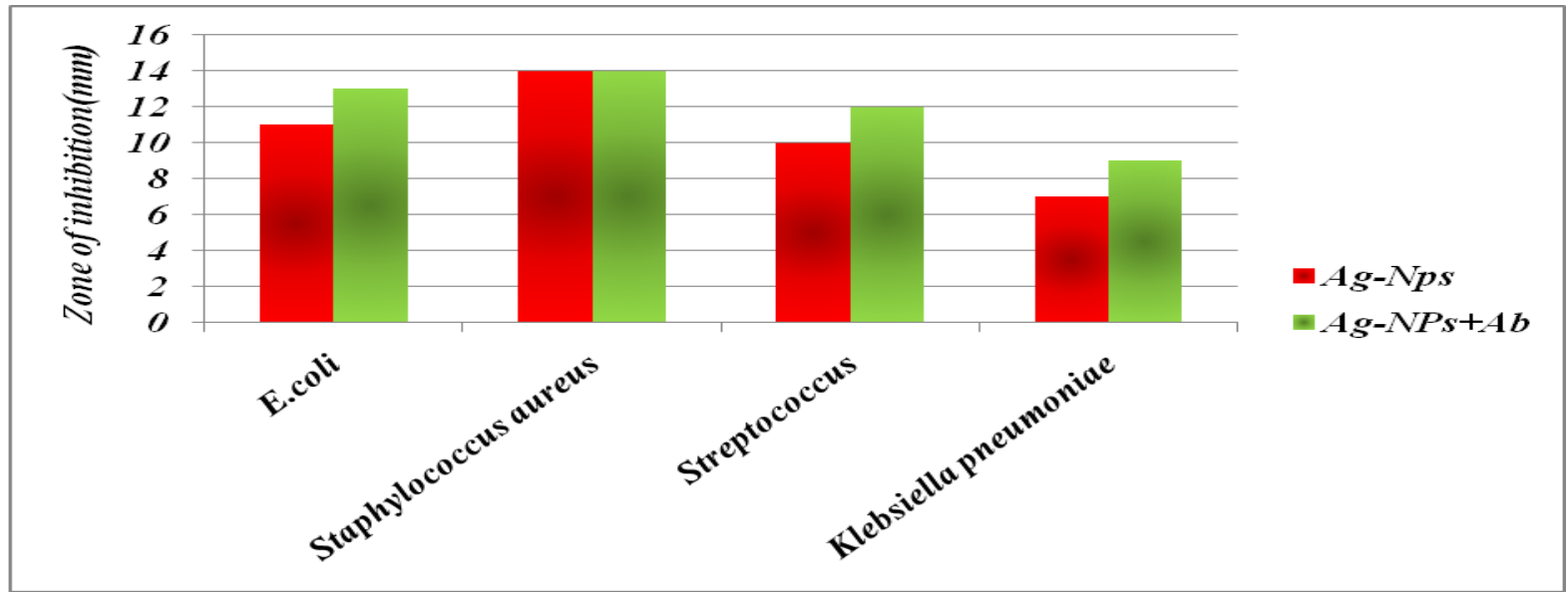




\section{Characterization by UV- Visible Spectroscopy}

Synthesized Ag-NPs absorption capacity was observed at every $24 \mathrm{hrs}$ of incubation. Figure 2 shows the absorption maxima $(0.72)$ band at $420 \mathrm{~nm}$ after 3 days of incubation. Up to some extent the $\mathrm{AgNO} 3$ intensity was increased with time was clearly recorded in the spectrum. The brownish yellow color is due to the "surface of plasmon resonance of deposited silver nanoparticles" that is, "the color of the AgNPs due to the coherent and collective oscillations of the surface electrons" (Link, 2003). The peak formed at $420 \mathrm{~nm}$ is the characteristic indication for the presence of the proteins and enzymes. These bioactive compounds are responsible for the reduction of metal ions for synthesis of nanoparticles (Wiley et al., 2006).

\section{FTIR Analysis of Ag-NPs}

Silver nanoparticles were analyzed through FTIR to find out the interactions between silver and bioactive compounds produced by fungi. These bioactive compounds play major role in metal ion reduction, stabilization and synthesis of Ag- NPs. Spectra shows the transmission peak at 3383, 2361, 1723, 1581, 1389, 1112, 616. Similarly transmission peak for the fungus Aspergillus niger containing silver nano particles were obtained at $3383,2361,1723$, 1581, 1389, 1112, 616. The absorption peaks 3383 corresponds to Amide $\mathrm{C}=\mathrm{O}$ and Amine groups like wise 2361 corresponds to acid O-H, 1723 to Aldehyde, Ketone, Ester $\mathrm{C}=\mathrm{O}, 1581$ to Aromatic $\mathrm{C}=\mathrm{C}, 1389$ to $\mathrm{C}-\mathrm{O}$, 1112 to Ketones C-C stretch and 616 to Chloroaklanes C-C1 stretch. Thus the result indicates that the amide, amine, alkane, aldehyde, carboxylic acid, alkyne groups of Aspergillus niger are mainly involved in the fabrication of silver nano particles.
Antibacterial Activity: The Ag-NPs produced from the Aspergillus niger were assayed for their antibacterial activity with and without the presence of standard antibiotic $(\mathrm{Ab})$ chloramphenicol against the Escherichia coli, Klebsiella pneumonia, Bacillus cereus, Staphylococcus aureus and Streptococcus sp. A.niger is good efficient in production and green synthesis of nanoparticles. Maximum zone of inhibition was observed with Staphylococcus aureus $(14 \mathrm{~mm})$, and there is no significant difference observed with Ag- NPs + Ab, remaining bacterial species showed difference in zone of inhibition formed with $\mathrm{Ag}-\mathrm{NPs}+\mathrm{Ab}$. The diameter of zones of inhibition (mm) formed by $E$. coli, $K$. pneumonia, S. aureus and Streptococcus sp., showed the similarities with Manju bala,Vedpriya arya and Mudasir et al., (2013). The unusual salient features of AgNPs make them being perfect model for various technologies including the antimicrobial, optical and biomedical hygiene applications, as well as use in nanotoxicology studies.

In conclusion, the filamentous fungus, $A$. niger has shown potential for extracellular synthesis Ag-NPs. Synthesis of Ag-NPs using the cell free filtrate is rapid. This indicates nanoparticle synthesis from biological process is quick suitable for larger scale production. The characterization of Ag-NPs was through UV visible spectrophotometer and FTIR analysis. Nanotechnology exhibits contemporary and revolutionary approach to formulate and to test the new approaches based on antimicrobial properties from the metallic nanoparticles. Ag-NPs showed remarkable antibacterial activity against Escherichia coli, Klebsiella pneumonia, Staphylococcus aureus and Streptococcussp. The bacteria which showed the resistant to antibiotics, indicates sensitivity to Abs in combination 
with silver nanoparticles. Synthesis of AgNPs using $A$. niger is potential in, eliminating the problems caused by chemicals that produce negative effects in applications, this results biological synthesis of nanoparticles are more biocompatible.

\section{References}

Ahmad, A., P. Mukherjee, S. Senapati, D. Mandal, M.I. Khan, R. Kumar, M. Sastry. 2003. Extracellular biosynthesis of silver nanoparticles using the fungus Fusarium oxysporum. Colloids Surf. B., 28: 313.

Bhainsa, K.C. and D'Souza, S.F. 2006. "Extracellular biosynthesis of silver nanoparticles using the fungus Aspergillus fumigatus", Colloids Surf. B. Biointerfaces, Vol. 47, pp. 160164.

Bhushan, B. 2004. in Springer Handbook of Nanotechnology, Spinger-Verlag Berlin, Heidelberg.

Binupriya, A., Sathishkumar, M., Yun, S. 2010. Biocrystallization of silver and gold ions by inactive cell filtrate of Rhizopus stolonifer. Colloid Surf B., 79: 531-534.

Gong, P., Li, H., He, X., Wang, K., Hu, J., Tan, W., Tan, S., Zhang, X.Y. 2007. Preparation and antibacterial activity of Fe3O4@Ag nanoparticles. Nanotechnol., 18: 604-611.

Ingle, A., Gade, A., Pierrat, S., Sonnichsen, C., and Rai, M. 2008. Mycosynthesis of Silver Nanoparticles Using the Fungus Fusarium acuminatum and its Activity Against Some Human Pathogenic Bacteria, Curr. Nanosci., 4: 141-144.

Link, S., and El- Sayed, M.A. 2003. Optical properties and ultrafast dynamics of nanocrystals, Annu. Rev. Phys. Chem., 53: 331 .
Manju bala and Vedpriya arya. 2013. Biological synthesis of silver nanoparticles from aqueous extract of endophytic fungus Aspergillus fumigates and its antibacterial action. Int. J. Nanomaterials and Biostructures, 3(2): 37-41.

Mohanpuria, P., Rana, N.K., Yadav, S.K. 2008. Biosynthesis of nanoparticles: Technological concepts and future applications. J. Nanopart. Res., 10: 507-517.

Mudasir, A., Dar, Avinash Ingle, Mahendra Rai. 2013. Enhanced antimicrobial activity of silver nanoparticles synthesized by Cryphonectria sp. evaluated singly and in combination with antibiotics. Nanomed. NBM, 9: 105-110.

Narayanan, K.B., Sakthivel, N. 2010 Biological synthesis of metal nanoparticles by microbes. $A d v$. Colloid Interface Sci., 156: 1-13.

Petit, C., Lixon, P., Pileni, M.P. 1993. In situ synthesis of silver nanocluster in AOT reverse micelles. $J$. Physical Chem., 97(49): 12974-12983.

Pol, V.G., D.N. Srivastava, O. Palchik, V. Palchik, M.A. Slifkin, A.M. Weiss, and and A. Gedanken. 2002. Sonochemical Deposition of Silver Nanoparticles on Silica Spheres. Langmuir, 18(8): 3352-3357.

Prameela Devi, T., Kulanthaivel, S., Deeba Kamil, Jyothi Lekha Borah, Prabhakaran, N., and Srinivasa, N. 2013. Biosynthesis of nanoparticles from Trichoderma species. Indian $J$. Experimental Biol., 543-547.

Rai, A., A. Singh, A. Ahmad, M. Sastry. 2006. Langmuir Role of Halide Ions and Temperature on the Morphology of Biologically Synthesized Gold Nanotriangles, 22: 736.

Ravendran, P., Fu, J., Wallens, L., Am, J., Virender, K., Sharma and Ria, A. 
2003 Silver nanoparticles: Green synthesis and their antimicrobial activities, Chem. Soc., M125, 13940.

Sathishkumar, M., K. Sneha, S.W. Won, C.W. Cho, S. Kim, Y.S. Yun. 2009. Colloids Surf, B Cinnamon zeylanicum bark extract and powder mediated green synthesis of nanocrystalline silver particles and its bactericidal activity, 73: 332 .

Shahverdi, R., S. Minaeian, H.R. Shahverdi, H. Jamalifar, A.A. Nohi. 2007. Proc. Biochem. Rapid synthesis of silver nanoparticles using culture supernatants of Enterobacteria: A novel biological approach, 42: 919.

Slawson, R.M., Van Dyke, M.I., Lee, H. and Trevor, J.T. 1992. Germanium and silver resistance, accumulation and toxicity in microorganisms. Plasmid, 27: 73.
Solanki, J.N. and Z.V.P. Murthy. 2010. Highly Monodisperse and Sub-Nano Silver Particles Synthesis Via Microemulsion Technique Colloids and Surfaces A: Physicochem. Engi. Aspects, 359: 31-38.

Wang, X.B., Cai, W.P., Lin, Y.X., Wang, G.Z. and Liang. C.H. 2010. Mass production of micro/nanostructured porous $\mathrm{ZnO}$ plates and their strong structurally enhanced and selective adsorption performance for environmental remediation. J. Mater. Chem., 20: 8582-8590.

Wiley, B.J., Im, S.H., McLellan, J., Siekkkinen, A., and Xia, Y. 2006. Maneuvering the surface Plasmon resonance of silver nanostructures through shape-controlled synthesis, $J$. Phy. Chem. B., 110: 15666.

\section{How to cite this article:}

Kalyani, P., K. Chandana Vineela, S. Geetha and Hemalatha, K.P.J. 2016. Green Synthesis of Silver Nano Particles from Aspergillus niger (MTCC-961). Int.J.Curr.Microbiol.App.Sci. 5(10): 50-56. doi: http://dx.doi.org/10.20546/ijcmas.2016.510.007 